\title{
Student conceptual resources for understanding mechanical wave propagation
}

\author{
Lisa M. Goodhew, ${ }^{1}$ Amy D. Robertson, ${ }^{2}$ Paula R. L. Heron, ${ }^{1}$ and Rachel E. Scherr ${ }^{2}$ \\ ${ }^{1}$ Department of Physics, University of Washington, 3910 15th Ave NE, Seattle, WA, 98195 \\ ${ }^{2}$ Department of Physics, Seattle Pacific University, 3307 3rd Ave W, Seattle, WA, 98119
}

\begin{abstract}
Here we present preliminary results of our investigation of introductory physics students' conceptual resources for understanding mechanical wave propagation. We analyzed a total of 446 student responses to a conceptual question about pulse propagation and identified two common, prevalent resources: (1) students treat pulses as macroscopic objects moving through a medium whose properties affect their speed, and (2) students treat pulses as propagating local disturbances. We illustrate some of the ways in which these resources manifested in student responses and discuss how we see such ideas as continuous with scientific understanding.
\end{abstract}

\section{INTRODUCTION}

Researchers' conceptualizations of pedagogical content knowledge - i.e., what instructors need to know in order to teach - include both knowledge of the specific content to be taught and knowledge of students' ideas (KSI) [1, 2]. Historically, the category of KSI in science has been framed in terms of student misconceptions or difficulties $[3,4]$ and research has contributed to instructors' KSI by identifying common, incorrect patterns in student reasoning (e.g., [5]). Our work expands the inventory, so to speak, of KSI by identifying some common, prevalent conceptual resources that students use to understand mechanical wave propagation.

The resources we report here are meant to support instructors in anticipating and taking up specific student ideas in their instruction, in the tradition of historical conceptualizations of KSI. They are also meant to illustrate how instructors can enact a view of their students' ideas as inherently sensible and potentially productive. Such a perspective has a number of documented benefits, including promoting students' conceptual understanding [6] and supporting student agency [7]. We take the perspective that even canonically incorrect ideas or ideas that have been reported as difficulties can be framed as resourceful. Because most of the literature on student ideas about wave mechanics (e.g., $[5,8,9])$ focuses on student difficulties, our work contributes both an alternative perspective and topic-specific KSI.

\section{THEORETICAL FRAMEWORK: RESOURCES}

We define a resource as an idea that could represent the beginning of more sophisticated scientific understanding [10-12]. Our orientation toward student thinking is consistent with that embodied in diSessa [13]: we assume that students' ideas represent ways of organizing experiences that are useful in some situations; that student thinking is often continuous with formal physics; and that quality learning builds on students' intuitive ideas about the physical world.

Theoretical accounts of resources depict knowledge as dynamic and context-dependent [13-15], such that resources that are activated in one context are not necessarily expected to be activated in another. In this paper, we report common, prevalent resources - those that come up in multiple versions of a given question, for many students. This choice is informed by our aim to provide instructors with a sense of what resources they might expect their students to use, in a typical introductory physics course. In the absence of a representative sample of introductory physics students, we draw on a model of generalizability that emphasizes recurrence - "how consistently a particular [pattern or relationship] reproduces across multiple sources of heterogeneity" [16]. This is consistent with a resources theoretical framework as long as we acknowledge the distinctive ways in which (and frequencies at which) these resources manifest for different students in different contexts [14, 15]. In short, our aims and theoretical framework lead us to frame the resources we report as those that may be likely to be activated in wave propagation contexts, and as illustrations of what it can look like to frame such ideas as productive.

\section{QUESTION DESIGN AND ADMINISTRATION}

We analyzed student responses to multiple versions of a single question about the propagation of a pulse along a string. The original question was drawn from Tongchai et al. [8], and is a modified version of a question from the Waves Diagnostic Test [17]. We chose this question because student difficulties in the context of this question have been well-documented $[8,17-19]$ and because we expect 
the phenomenon to be accessible to students with a range of lived experiences. Since we were interested in students' reasoning in addition to their answer choices, we modified the question to be freeresponse rather than multiple-choice.

After receiving an initial set of student responses, we made several modifications to the question to elicit students' reasoning about pulse speed and the relationship between pulse generation and pulse speed. All three versions of the pulse-flick question are shown in Figure 1.

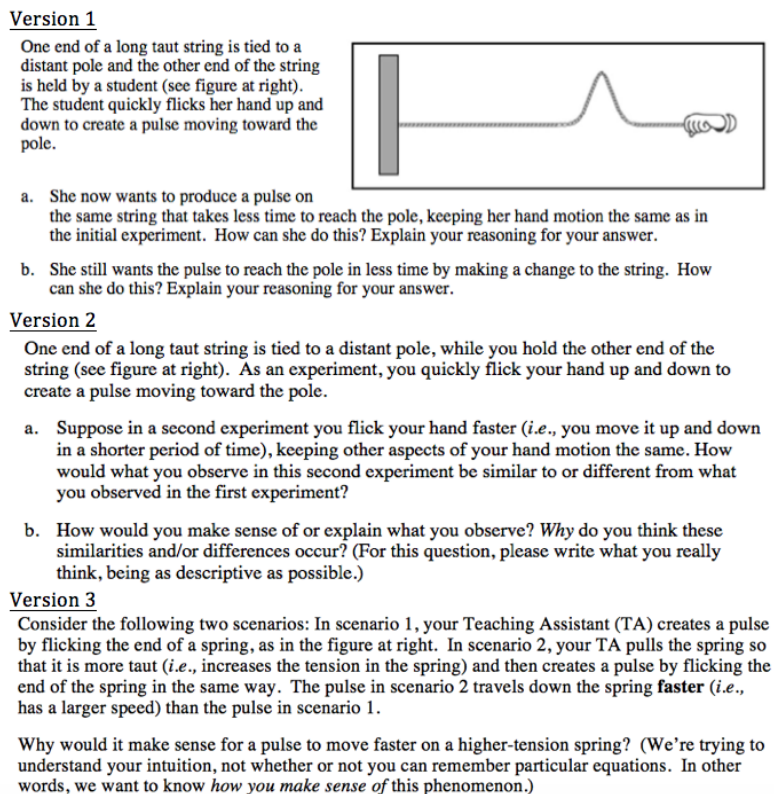
in a shorter period of time), keeping other aspects of your hand motion the same. How would what you observe in this second experiment be similar to or different from what

b. How would you make sense of or explain what you observe? Why do you think these similarities and/or differences occur? (For this question, please write what you really think, being as descriptive as possible.)

Version 3

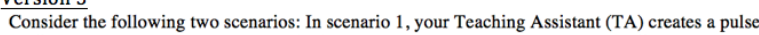
by flicking the end of a spring, as in the figure at right. In scenario 2, your TA pulls the spring so that it is more taut (i.e., increases the tension in the spring) and then creates a pulse by flicking the has a larger speed) than the pulse in scenario 1 .

Why would it make sense for a pulse to move faster on a higher-tension spring? (We're trying to understand your intuition, not whether or not you can remember particular equations. In other

words, we want to know how you make sense of this phenomenon.)

FIG. 1: The three versions of the pulse-flick question. V1 was modified from [8].

In total, 446 students were given one of the three versions of the pulse-flick question. These questions were given as a required part of the coursework in introductory physics courses at the University of Washington (UW), Seattle Pacific University (SPU), and California Polytechnic State University - San Luis Obispo (CPSLO). Table I provides additional details about the samples.

\section{DATA ANALYSIS}

Two authors (LMG and ADR) used student responses to create an emergent coding scheme [20] that depicted the resources that students were using to reason about propagation in the pulse flick question. For example, in the second student response discussed in Section V.B below, we identified the resourceful idea that the speed of the pulse is related to the force between particles of the string.
TABLE I: Administration of three versions of the pulse-flick question at multiple institutions.

\begin{tabular}{cccc}
\hline \hline Version & University & $\begin{array}{c}\text { Sample } \\
\text { size }\end{array}$ & $\begin{array}{c}\text { Fraction of course } \\
\text { participating }\end{array}$ \\
\hline 1 & UW & $\mathrm{N}=135$ & $86 \%$ \\
1 & CPSLO & $\mathrm{N}=68$ & $94 \%$ \\
2 & SPU & $\mathrm{N}=39$ & $75 \%$ \\
3 & $\mathrm{UW}$ & $\mathrm{N}=162$ & $70 \%$ \\
3 & $\mathrm{CPSLO}$ & $\mathrm{N}=42$ & $88 \%$ \\
\hline \hline
\end{tabular}

After LMG and ADR separately examined a subset of student responses from each version, all identified resourceful ideas were grouped according to our sense of their productive substance (e.g., thinking of the pulse as a propagating local disturbance). This produced a coding scheme with three codes, two of which were common across contexts and are discussed here.

LMG and ADR independently coded each student's response according to this scheme. A single response could be given more than one code to capture the full range of resources that a student appeared to be using. We compared the codes LMG and ADR separately assigned to each response and kept only codes that were assigned by both coders, such that the final codes assigned to a single response reflected $100 \%$ agreement between coders. From there, we calculated the percentage of student responses instantiating each resource. As a measure of percentage agreement, we took the normalized difference between the total number of codes possible and the total number of disagreements between the two coders, represented by:

$$
\frac{\left(n_{\text {possible codes }}\right)\left(n_{\text {coded responses }}\right)-\left(n_{\text {total disagreements }}\right)}{\left(n_{\text {possible codes }}\right)\left(n_{\text {coded responses }}\right)}
$$

For example, if a student's response was assigned codes 1 and 2 by the first coder and code 1 by the second, there would be 1 disagreement for this data set of one response. Since there are two possible codes, this would represent an percentage agreement of 0.50 . To the nearest hundredth, our percentage agreement for the entire data set was 0.82 .

\section{STUDENT RESOURCES FOR UNDERSTANDING MECHANICAL WAVES}

The resources we report here are those that were common and prevalent - i.e., those that were used 
by at least $20 \%$ of students in at least one context and that were elicited by multiple versions of the pulse-flick question at several universities. The prevalence each of these resources is detailed in Table II. In this section, we provide examples to illustrate each resource and discuss ways in which we see these resources as productive.

TABLE II: Prevalence of resources A and B in multiple contexts. Percentages represent the fraction of responses that both coders agreed instantiate resource A or B, respectively.

\begin{tabular}{lccccc}
\hline \hline \multirow{2}{*}{ Resource } & \multicolumn{4}{c}{ Prevalence } \\
\hline & \multicolumn{2}{c}{ Version 1 } & Version 2 & \multicolumn{2}{c}{ Version 3 } \\
& UW & CPSLO & SPU & UW & CPSLO \\
\hline A & $70 \%$ & $46 \%$ & $10 \%$ & $14 \%$ & $24 \%$ \\
B & $0 \%$ & $7 \%$ & $0 \%$ & $50 \%$ & $19 \%$ \\
\hline \hline
\end{tabular}

\section{A. Treating a pulse as an object moving through a medium whose properties affect its speed}

As shown in Table II, many responses to the pulse-flick question treated the pulse as a macroscopic object moving through a medium whose properties affect its speed. We assigned this code to responses that treated the pulse as a single object and described the effect that properties of the string had on its motion. For example: "Decreasing the mass density means less mass in the given length and as mass is a measurement of resisting inertia, the less mass the easier the acceleration" (V1). By implying that lower mass density means less resistance to the motion of the pulse, this student appropriately associates a change in the speed of the pulse with a change in the medium. Responses like this one often apply mechanics concepts to describe a mechanism by which this property affects the speed of the pulse. Another instantiation of this idea is: "With a lower density and the same energy a wave uses less energy to travel through the medium and more energy to 'create' its speed" (V3). By describing the pulse as using a certain amount of energy to move through a medium, this response implies thinking of the pulse as a macroscopic object. This student appropriately associates the speed of the pulse with the mass density of the spring and begins to explain the mechanism for its propagation using the concept of energy.

A different form of this resource is: "In a spring with less tension, a bit [of] slack will exist in the string. As the pulse travels, it will be slowed by that slack. So inversely, increasing the tension raises the speed." (V3). This response treats the pulse as an object that is impeded by the slack in the spring, and in doing so associates the pulse speed specifically with tension.

\section{B. Treating a pulse as a propagating local disturbance}

As shown in Table II, many responses treated the pulse as a propagating local disturbance of the medium. Responses that discussed motion of individual particles or small pieces of the string, described the propagation of the pulse as a series of events or the sequential movement of individual particles, or discussed how changing properties of the string or pulse affected individual particles of the string, received this code.

One form of this resource is the idea that if a point takes less time to move up and down, the pulse moves along the string faster. For example: "[If the amplitude is smaller] each part of the string doesn't have to go as high up and can rather have the wave propagate faster," (V1). This response treats the pulse as a propagating local disturbance of the string by focusing on the time it takes for each particle to move. We see this idea as a starting point for reasoning about the relationship between the speed of the pulse and the transverse motion of the string.

Other instances of this resource discuss forces between pieces of the string, such as: "It makes sense to me because as you increase the tension you increase the force in the string so the force that each particle can exert on the one next to it is larger" (V3). This response implies that the motion of the pulse depends on each particle of the string pulling the next up and down, so if one particle exerts a greater force on the next, the pulse travels faster. As another example: "The wave pulse speed would increase when the tension of the spring increases because higher tension means there's a higher force between the particles in the string. When the wave is displaced, there is a greater restoring force to bring the particles back to equilibrium. Therefore, there's a greater tendency to pass displacement along, resulting in a higher wave speed," (V3). In referencing the force between particles and the tendency to "pass displacement along," this response implies a nascent mechanism for pulse propagation. Responses such as these are resourceful because they show students connecting pulse speed with important mechanics concepts such as force and acceleration and require thinking of the motion of the pulse in a fundamental, physical way. 


\section{DISCUSSION}

The intent of this work is to inform instruction on mechanical waves by identifying and illustrating some of the conceptual resources that instructors might expect their students to use. To this end, we have described two common conceptual resources that students used to reason about mechanical wave propagation, contributing to instructors' repertoire of KSI. In keeping with our theoretical framework, which highlights the contextdependence of student thinking, we show that these resources do not necessarily manifest in the same way for every student or in all contexts. For example, we show that resource B can be instantiated in ideas about the transverse speed of a segment of the string or in ideas about the forces between particles in the string. In discussing these particular responses, we have illustrated how we enact our orientation toward students' thinking.

Our analysis suggests that in certain contexts, student ideas that have historically been reported as difficulties may be productive. Wittmann reports that treating a pulse as a Newtonian particle can lead to difficulties in the context of pulse generation [19] - particularly the incorrect idea that flicking the string faster makes the pulse move faster, which has been reported by several authors [9, 17]. This kind of thinking could be categorized as resource A, according to our scheme. We argue that treating a pulse as a macroscopic object can be productive when used to reason about pulse propagation.

As is evident in Table II, the prevalence of resources $\mathrm{A}$ and $\mathrm{B}$ varies with question context. This is consistent with the theoretical framework outlined above and it suggests that there may be spe- cific questions that elicit particular resources. It also raises questions as to what may be influencing the variability of student responses. Though we do not have enough data to answer this question, possibilities include instructional approach, epistemological framings (e.g., do students see themselves as sense-making or answer-making), or differences in student populations. Our continued work will explore the extent to which particular questions reliably elicit certain resources or a diversity of resources, further contributing to instructor KSI.

Though PER has used research on student thinking to develop and validate instructional strategies and materials, many of these are based on studies of student difficulties or misconceptions. Far fewer materials and strategies focus on potentiallyproductive ideas that students bring to bear in learning physics. We suspect that one reason is that, particularly in a recurrence-oriented field such as physics, instructors want to know what resources they can expect their students to use and that resources-oriented instruction is effective in improving students' conceptual understandings. Both are concerns that research has not yet spoken to. This work lays a foundation for developing resources-oriented instructional materials, testing these materials on a large scale, and providing instructors with the KSI necessary to enact resourcesoriented instruction.

\section{Acknowledgments}

This work was supported in part by NSF grants 1608510 and 1256082. We wish to thank instructors at the UW, SPU, and CPSLO for asking our questions in their courses.
[1] D.L. Ball, M.H. Thames, and G. Phelps, J. Teach. Educ. 59, 389 (2008).

[2] L. Shulman, Harv. Educ. Rev. 57, 1 (1987).

[3] E. Etkina, Phys. Rev. ST PER 6, 020110 (2010).

[4] J.R. Thompson, W.M. Christensen, and M.C. Wittmann, Phys. Rev. ST PER 7, 010108 (2011).

[5] M. Kryjevskaia, M. R. Stetzer, and P. R. L. Heron, Am. J. Phys. 80, 339 (2012).

[6] T.P. Carpenter et. al., Am. Educ. Res. J. 26, 499 (1989).

[7] J.E. Coffey et. al., J. Res. Sci. Teach. 48, 1109 (2011).

[8] A. Tongchai, et al., Phys. Rev. ST PER 7, 1 (2011).

[9] E. M. Kennedy and J. R. De Bruyn, Can. J. Phys. 89, 1155 (2011).

[10] D. Hammer, Am. J. Phys. 68, S52 (2000).

[11] D. Hammer, F. Goldberg, and S. Fargason, Rev. Sci.
Math. ICT Educ. 6, 51 (2012).

[12] B. W. Harrer, V. J. Flood, and M. C. Wittmann, Phys. Rev. ST PER 9, 1 (2013).

[13] A. diSessa, Cognition \& Instruction 10, 105 (1993).

[14] D. Hammer et al., in Transfer of Learning from a Modern Multidisciplinary Perspective ed. J.P. Mestre (Information Age Publishing, Greenwich, 2005), pp. 89-119.

[15] R.E. Scherr, Am. J. Phys. 75, 272 (2007).

[16] T.D. Cook, Educ. Eval. Policy Anal. 24, 175 (2002).

[17] M. C. Wittmann, University of Maryland, 1998.

[18] M. C. Wittmann, R. N. Steinberg, and E. F. Redish, Int. J. Sci. Educ. 25, 23 (2003).

[19] M. Wittmann, Int. J. Sci. Educ. 24, 97 (2002).

[20] Krippendorff, K. (2013). Content Analysis: An Introduction to Its Methodology (3rd ed.). Thousand Oaks, CA: Sage. 